\title{
Clinical Effects of Safflower Pill of Dedu Seven Medicinal Herbs Plus Adefovir Dipivoxil on Liver Fibrosis Resulting from Chronic Viral Hepatitis B
}

\author{
Yi Na ${ }^{1}$, Siwen Wei ${ }^{1}$, Xiaoran Wang ${ }^{1}$, Yi Wang ${ }^{2}$, Ruilian $\mathrm{Ma}^{2}$, Lidao Bao ${ }^{2, *}$ \\ ${ }^{1}$ College of Pharmacy, Inner Mongolia Medical University, Hohhot, P. R. China \\ ${ }^{2}$ Department of Pharmacy, Affiliated Hospital of Inner Mongolia Medical University, Hohhot, P. R. China
}

Email address:

baolidao237@163.com (Lidao Bao)

*Corresponding author

\section{To cite this article:}

Yi Na, Siwen Wei, Xiaoran Wang, Yi Wang, Ruilian Ma, Lidao Bao. Clinical Effects of Safflower Pill of Dedu Seven Medicinal Herbs Plus Adefovir Dipivoxil on Liver Fibrosis Resulting from Chronic Viral Hepatitis B. International Journal of Biomedical Science and Engineering. Vol. 5, No. 3, 2017, pp. 24-28. doi: 10.11648/j.ijbse.20170503.12

Received: April 17, 2017; Accepted: May 18, 2017; Published: June 6, 2017

\begin{abstract}
To study the clinical effects of Safflower Pill of Dedu Seven Medicinal Herbs combined with Adefovir Dipivoxil on liver fibrosis resulting from chronic viral hepatitis B.116 patients with chronic hepatitis B fibrosis were randomly divided into two groups ( $\mathrm{n}=58$ for each group): treatment group (Safflower Pill of Seven Medicinal Herbs combined with Adefovir Dipivoxil) and control group (Adefovir Dipivoxil) $(\mathrm{n}=58)$ for clinical observation of 24 weeks. After 24 weeks, the effective rates were $93.10 \%$ and $81.03 \%$ in the treatment group and control group respectively; the indexes of liver function, liver fibrosis and radiographic indexes of the patients in both groups were significantly decreased $(\mathrm{P}<0.05$ or $\mathrm{P}<0.01)$; The differences between the two groups were statistically significant $(\mathrm{P}<0.05)$. The effect of Safflower Pill of Seven Medicinal Herbs combined with Adefovir Dipivoxil for the treatment of chronic hepatitis B fibrosis is more effective than that of Adefovir Dipivoxil alone.
\end{abstract}

Keywords: Adefovir Dipivoxil, Chronic Viral Hepatitis B, Liver Fibrosis, Safflower Pill of Seven Medicinal Herbs

\section{Introduction}

Liver fibrosis is an inevitable pathological process that chronic hepatitis B (CHB) develops to cirrhosis. If liver fibrosis can be blocked, alleviated or even reversed, the prognosis of hepatopathy can be greatly improved to a large extent. [1] Therefore, it is of greatly clinical significance for anti-fibrotic therapy to delaying and reversing liver fibrosis and preventing the occurrence and development of cirrhosis. [2] The etiological treatment of anti-fibrotic therapy by traditional medicine combined with antiviral therapy by western medicine is a relatively effective method for the treatment of chronic hepatitis B fibrosis. [3] Safflower Pill of Dedu Seven Medicinal Herbs is a commonly used prescription in Mongolian treatment of liver diseases, which is composed of seven Mongolian medicines, i.e. Safflower, Ephedra, Gypsum, Manshuriensis, Chinese Violet, Myrobalan and Flos Scabiosae, which shows the efficacies of detoxication, removing blood heat from the liver, conditioning physical quality and eliminating jaundice, and has a significant effect in improving symptoms and signs of chronic hepatitis, protecting liver and lowering transaminase and conversion of HBV markers. [4] In this study, the Mongolian medicine Safflower Pill of Seven Medicinal Herbs and the Western medicine Adefovir Dipivoxil were applied in the treatment of 116 patients with chronic hepatitis $\mathrm{B}$ fibrosis to observe the effects by grouping method. The results were reported as follows.

\section{Materials and Methods}

\subsection{General Information}

This study has been approved by the ethics committee of Affiliated Hospital of Inner Mongolia Medical University. Consent has been obtained before examination and treatment 
for enrolled patients according to the Declaration of Helsinki and relevant laws in China. All treatments were performed based on the patients' best interests. All the 116 patients enrolled from January 2010 to June 2012 were randomly divided into 2 groups. The treatment group consisted of 42 cases: 42 males, 16 females; 23-59 years old (average: $31.81 \pm 6.24$ ); 2-19.1 years of disease course (average: $6.34 \pm 3.02)$. The control group consisted of 58 cases: 43 males, 15 females; 23-59 years old (average: $32.19 \pm 6.65$ ); 2.2-18.7 years of disease course (average: 5.81 \pm 2.32 ). The data of the two groups did not differ significantly $(\mathrm{P}>0.05)$.

\subsection{Inclusion Standards}

\subsubsection{Diagnosis Standards}

All the cases met with the standards of the Guideline for Prevention and Treatment of Chronic Hepatitis $B$ compiled by the Chinese Society of Hepatology, Chinese Medical Association in 2005 [5] and the Guidelines for Diagnosis and Treatment of Liver Fibrosis in Integrative Medicine Practice approved by the Liver Disease Committee, Chinese Association of Integrative Medicine in 2006. [6] (1) The medical history of chronic hepatitis B is more than 6 months, with serum HBsAg positive, $2 \times \mathrm{ULN} \leq \mathrm{ALT} \leq 4 \times \mathrm{ULN}$; (2) serum indexes of liver fibrosis: at least two items in HA, PCIII, CIV and LN rise abnormally; (3) through Bultrasound examination, the patients are found rough liver capsule, densificated, thickened and enhanced intrahepatic echo with uneven distribution, unclear vascular orientation, or portal vein diameter widening and spleen thickening.

Mongolian medicine diagnosis standards

The disease was determined to be the syndrome of liver depression and blood stasis with reference to the diagnostic criteria of Mongolian medicine syndrome in the Guiding Principles for Clinical Study of New Chinese Medicines (2002). [7] Its main symptoms include abdominal distension, stabbing or distending pain in lateral thorax; secondary symptoms consist of loss of appetite, malaise, fatigue, hepatosplenomegaly, gloomy complexion, liver palms, spider angioma, dark purple tongue or ecchymosis, petechiae, stringy or sluggish pulse.

\subsubsection{Exclusion Standards}

(1) Serum TBIL $>51.3 \mu \mathrm{mol} / \mathrm{L}$ or PTA $<60 \%$; (2) patients receiving anti-fibrotic or antiviral pharmacotherapy in the last six months; (3) patients with hepatopathy or liver cancer caused by drug, immunity, heredity and other reasons; (4) pregnant and lactating women; (5) patients suffering from severe cardiovascular and cerebrovascular diseases and unstable diabetes; (6) patients with superinfection of HAV, $\mathrm{HCV}$ and HEV.

\subsection{Treatment Methods}

The treatment group was treated with Safflower Pill of Seven Medicinal Herbs orally (Ingredients: Safflower, Ephedra, Gypsum, Manshuriensis, Chinese Violet, Myrobalan and Flos Scabiosae, produced by Inner Mongolia Kulun Mongolia Pharmaceutical Factory, Batch No.:
Z15020875), 1 to 3 times daily with 2.5 to $5 \mathrm{~g}$ once; plus Adefovir Dipivoxil (Hepsera, produced by GlaxoSmithKline Plc) 10mg orally, s.i.d. The control group just orally administrated the Western medicine Adefovir Dipivoxil (Hepsera, produced by GlaxoSmithKline Plc) $10 \mathrm{mg}$, s.i.d. The course of treatment was 24 weeks, during which other anti-fibrotic and antiviral drugs were forbidden to use.

\subsection{Observation Items}

\subsubsection{Efficacy Indicators}

Liver fibrosis indexes: radioimmunoassay was adopted for the detection of serum hyaluronic acid (HA), procollagen type III (PC III), collagen type IV (CIV) and laminin (LN); radiographic indexes: spleen length diameter, spleen thickness, inner diameter of portal vein; liver function, HBVDNA, five indexes of hepatitis B (HBV-DNA and five indexes of hepatitis B were tested by ELISA, and HBV-DNA by Fluorescent PCR); clinical symptoms and signs.

\subsubsection{Observation Timing}

Liver fibrosis indexes, radiographic indexes, HBV-DNA hepatitis B markers, ECG, liver and kidney functions, feces and urine routine were examined once at the time of being selected and at the end of the experiment respectively; liver function and blood routine were detected once respectively at the time of being selected, in the first, third and fifth months and at the end of the experiment; clinical symptoms and signs were examined once monthly, with adverse reactions recorded. The measurement of all the indicators was performed by the Laboratory Department of Attached Hospital of Henan Institute of Chinese Traditional Medicine (Henan Province).

\subsection{Treatment Efficacy Evaluation Standards}

The standards were prepared in accordance with the Guidelines for Diagnosis and Treatment of Liver Fibrosis in Integrative Medicine Practice. [6] Markedly effective: the measured values of at least two serological markers of liver fibrosis (HA, PC III, CIV and LN content) decline by larger than or equal to $50 \%$ compared with those before the treatment or return to normal; serum liver function indicators basically return to normal, and clinical symptoms and signs are significantly improved. Effective: the measured values of any two serological markers of liver fibrosis (HA, PC III, CIV and LN content) decreased by larger than or equal to $25 \%$ compared with those before the treatment; serum liver function indicators, clinical symptoms and signs improve. Ineffective: the cases can not meet the effective standards. Effective rate $=$ (markedly effective case number + effective case number) $\mathrm{n} \times 100 \%$.

\subsection{Statistical Analysis}

All data were analyzed by SPSS 18.0. The measurement data were subjected to the $\mathrm{t}$ test, and the numeration data were subjected to the $\chi^{2}$ test. $\mathrm{P}<0.05$ was considered statistically significant. 


\section{Results}

\subsection{Comprehensive Efficacy Comparison}

The effective rates of the treatment group and the control group were $93.10 \%$ and $81.03 \%$ respectively, with the difference statistically significant $(\mathrm{P}<0.05)$.

\subsection{Effect of Treatment on Liver Fibrosis Indexes}

The difference in serum liver fibrosis indexes before the treatment was not statistically significant between the two groups $(\mathrm{P}>0.05)$, which was comparable. In the treatment group, there was significant difference in serum liver fibrosis indexes before and after the treatment $(\mathrm{P}<0.01$ or $\mathrm{P}<0.05)$. In the control group, the difference in serum liver fibrosis indexes was statistically significant before and after the treatment $(\mathrm{P}<0.05)$. By comparing HA, PC II, LN and CIV between the two groups after the treatment, the difference had statistical significance $(\mathrm{P}<0.01)$ (Table 1$)$.

Table 1. Liver fibrosis indexes before and after treatment $(\bar{x} \pm s, \rho / \mathrm{mg} / L)$.

\begin{tabular}{|c|c|c|c|c|}
\hline \multirow{2}{*}{ Item } & \multicolumn{2}{|c|}{ Treatment $(n=58)$} & \multicolumn{2}{|l|}{ Control $(n=58)$} \\
\hline & Before & After & Before & After \\
\hline HA & $258.56 \pm 68.07$ & $123.37 \pm 43.52^{*} \Delta \Delta$ & $259.12 \pm 70.12$ & $189.79 \pm 46.56^{\#}$ \\
\hline PCIII & $214.47 \pm 54.49$ & $142.59 \pm 47.14^{\# \Delta}$ & $215.43 \pm 50.31$ & $169.31 \pm 46.23^{\#}$ \\
\hline LN & $149.35 \pm 56.10$ & $115.32 \pm 47.35^{\#} \Delta$ & $150.21 \pm 56.33$ & $125.28 \pm 48.19^{\#}$ \\
\hline CIV & $176.13 \pm 40.92$ & $131.23 \pm 30.87^{\#} \Delta$ & $175.96 \pm 41.17$ & $148.34 \pm 36.16^{\#}$ \\
\hline
\end{tabular}

Compared to the same group before treatment: \#P<0.05, ${ }^{*} \mathrm{P}<0.01$; compared to the control group after treatment: $\Delta \mathrm{P}<0.05, \Delta \Delta \mathrm{P}<0.01$.

\subsection{Effect of Treatment on Radiographic Indicators}

In terms of inner diameter of portal vein, spleen thickness and spleen length diameter, the difference was statistically significant in the treatment group before and after treatment
$(\mathrm{P}<0.05)$; the difference had statistical significance in the control group before and after the treatment $(\mathrm{P}<0.05)$; there was significant difference between the two groups after the treatment $(\mathrm{P}<0.05)$ (Table 2).

Table 2. Radiographic indicators before and after treatment $(\bar{x} \pm s)$.

\begin{tabular}{|c|c|c|c|c|}
\hline \multirow{2}{*}{ Item } & \multicolumn{2}{|c|}{ Treatment $(n=58)$} & \multicolumn{2}{|c|}{ Control $(n=58)$} \\
\hline & Before & After & Before & After \\
\hline Inner diameter of portal vein $(\mathrm{L} / \mathrm{mm})$ & $14.6 \pm 1.4$ & $10.4 \pm 1.5^{\Delta}$ & $14.7 \pm 1.4$ & $11.5 \pm 1.7^{\#}$ \\
\hline Spleen thickness $(\mathrm{L} / \mathrm{cm})$ & $4.16 \pm 0.79$ & $3.55 \pm 0.45^{\#} \Delta$ & $4.11 \pm 0.82$ & $3.70 \pm 0.63^{\#}$ \\
\hline Spleen length diameter $(\mathrm{L} / \mathrm{cm})$ & $13.43 \pm 2.29$ & $10.50 \pm 1.82^{\# \Delta}$ & $13.51 \pm 2.91$ & $11.71 \pm 1.85^{\#}$ \\
\hline
\end{tabular}

Compared to the same group before treatment: \#P<0.05; compared to the control group after treatment: $\Delta \mathrm{P}<0.05$.

\subsection{Effect of Treatment on Liver Function Indicators}

The liver function indicators of the two groups were significantly improved before and after the treatmen $(\mathrm{P}<0.05$

or $\mathrm{P}<0.01)$; the difference had statistical significance between the two groups after the treatment $(\mathrm{P}<0.05)$ (Table 3$)$.

Table 3. Liver function indicators before and after treatment $(\bar{x} \pm s)$.

\begin{tabular}{|c|c|c|c|c|}
\hline \multirow{2}{*}{ Item } & \multicolumn{2}{|c|}{ Treatment $(n=58)$} & \multicolumn{2}{|l|}{ Control $(n=58)$} \\
\hline & Before & After & Before & After \\
\hline ALT (U/L) & $121.01 \pm 32.95$ & $34.49 \pm 13.81 * \Delta$ & $120.13 \pm 33.87$ & $54.52 \pm 21.34^{\#}$ \\
\hline AST (U/L) & $82.21 \pm 21.36$ & $30.63 \pm 11.57^{\#} \Delta$ & $81.97 \pm 19.75$ & $42.19 \pm 10.05^{\#}$ \\
\hline TBIL $(\mu \mathrm{mol} / \mathrm{L})$ & $40.15 \pm 10.23$ & $15.36 \pm 9.02^{\#} \Delta$ & $40.38 \pm 9.87$ & $20.79 \pm 7.12^{\#}$ \\
\hline $\operatorname{ALB}(\rho / \mathrm{mg} / \mathrm{L})$ & $34.54 \pm 7.28$ & $42.67 \pm 2.43^{\# \Delta}$ & $34.71 \pm 7.30$ & $34.99 \pm 3.14^{\#}$ \\
\hline
\end{tabular}

Compared to the same group before treatment: \#P<0.05, ${ }^{*} \mathrm{P}<0.01$; compared to the control group after treatment: $\Delta \mathrm{P}<0$. 05 .

\subsection{Effect of Treatment on Five Indexes of Hepatitis $B$ and $H B V-D N A$}

There was significant difference between the two groups before and after the treatment in the negative conversion rates of $\mathrm{HBeAg}$ and $\mathrm{HBV}-\mathrm{DNA}(\mathrm{P}<0.05)$, but the difference was not significant between the two groups after the treatment $(\mathrm{P}>0.05)$ (Table 4).

Table 4. Negative conversion rates of HBeAg and $H B V$-DNA before and after treatment (\%).

\begin{tabular}{|c|c|c|c|c|}
\hline \multirow{2}{*}{ Item } & \multicolumn{2}{|c|}{ Treatment $(\mathrm{n}=58)$} & \multicolumn{2}{|c|}{ Control $(n=58)$} \\
\hline & Before & After & Before & After \\
\hline HBsAg negative conversion rate & 0 & 0 & 0 & 0 \\
\hline $\mathrm{HBeAg}$ negative conversion rate & 62 & $37^{\# \boldsymbol{\Lambda}}$ & 59 & $41^{\#}$ \\
\hline HBV DNA negative conversion rate & 100 & $81^{\# \boldsymbol{\Lambda}}$ & 100 & $75^{\#}$ \\
\hline
\end{tabular}

Compared to the same group before treatment: $\# \mathrm{P}<0.05$; compared to the control group after treatment: $\mathbf{\Delta} \mathrm{P}>0.05$. 


\section{Discussion}

Liver fibrosis is a complex pathological process with initiative progress and dynamic changes, [8] involving multiple aspects and factors. All the stages of occurrence and development of liver fibrosis should be taken into account in treatment strategy, including treating primary diseases or removing etiological factors, eliminating hepatic inflammation, inhibiting formation of collagen fibres and promoting collagen degradation, etc. [9] Therefore, in order to reach the best clinical effect, comprehensive measures should be taken in the treatment of chronic hepatitis B fibrosis. [10]

Chronic persistent or active hepatitis can be attributed to the category of "hepatopyrexia" and "hepatomegaly" in the Mongolian medicine. [11] Mongolian medicine believes that the disease is caused by long-time stagnation for pathogenic heat invading the liver, which is induced by three roots disorder, nutrient substance indigestion and liver dysfunction. It is manifested clinically with general malaise, feeling body heavy and fatigue, poor appetite, loss of appetite, bitter taste, abdominal distension, right upper abdominal pain and liver pain and wasting away, [12] which should be treated based on the principle of removing blood heat from the liver and detoxification. Safflower Pill of Seven Medicinal Herbs is a Mongolian medicine with the effects of detoxication, removing blood heat from the liver, conditioning physical quality and eliminating jaundice, as well as helping digestion and nourishing the liver, and its effect on main pathogenesis of chronic hepatitis has been confirmed. [13] Previous experiments have proved that the Safflower Pill of Seven Medicinal Herbs has obvious effects of protecting liver and lowering transaminase for CCI4-induced acute liver injury in mice, and can significantly improve lesion and necrosis of liver tissue and degeneration. [14] Clinical studies have confirmed that the Safflower Pill of Seven Medicinal Herbs can alleviate liver inflammatory response, promote albumin synthesis and metabolism of bilirubin, protect liver cells; regulate metabolism of extracellular matrix (mainly collagen); improve partial microcirculation in liver and spleen, reduce portal pressure, prevent bleeding and other anti-fibrotic effects. [15]

Antiviral therapy is also one of the important means to delay and reverse liver fibrosis induced by chronic hepatitis B. [16] Adefovir Dipivoxil, as an antiviral agent of nucleoside commonly used in clinic, has a strong role in inhibiting HBVDNA replication, [17] and it can reduce liver inflammation, promote the recovery of liver function and prevent liver fibrosis progression, and its resistance rate is low. [18] In 2007, in the CBH Prevention Guide proposed by the American Association for the Study of Liver Diseases (AASID), moderate to severe inflammation or obvious liver fibrosis shown by liver biopsy is recommended as one of the indications of antiviral therapy. [19] Many clinical studies have further confirmed that antiviral therapy in combination with anti-fibrotic drug therapy can promote the reverse of liver fibrosis. [20]
In this study, the effects of Safflower Pill of Seven Medicinal Herbs combined with Adefovir Dipivoxil on chronic hepatitis B fibrosis were superior than those of Adefovir Dipivoxil alone in improving symptoms and fibrosis or even reversing fibrosis, and the resistance rate of Adefovir Dipivoxil was lower than that of Lamivudine. Under the guidance of Mongolian medical theory, we selected heat-clearing and detoxifying drugs clinically. The Safflower Pill of Seven Medicinal Herbs has the effects of clearing liver heat, detoxification and physical conditioning. In this prescription, Safflower that can remove heat from the liver and activate blood circulation is the basic remedy, supplemented by Ephedra with the effect of clearing liver heat and stopping bleeding, and Gypsum removing heat and jaundice, which is assisted and guided by Manshuriensis, Flos Scabiosae and Chinese Violet to clear liver heat and Myrobalan to relieve liver heat, detoxify and condition physical quality. The experimental results can prove that the Safflower Pill of Seven Medicinal Herbs combined with Adefovir Dipivoxil is an effective therapeutic regimen integrated Mongolian medicine with Western medicine for liver fibrosis resulting from chronic hepatitis B. In addition, no significant adverse reactions have been found yet in the combined application clinically.

\section{Acknowledgement}

This study was financially supported by the National Natural Science Founda-tion of China (grant no. 81550047, for LDB). The Nature Science Foundation of Inner Mongolia Autonomous Region (2015 MS (LH) 0805 for YW; 2013MS1224, for LDB; http://nsbr.nmkjt.gov.cn/index.aspx).

\section{References}

[1] Andersen ES, Ruhwald M, Moessner B, Christensen PB, Andersen O, Eugen-Olsen J, et al. Twelve potential fibrosis markers to differentiate mild liver fibrosis from cirrhosis in patients infected with chronic hepatitis $\mathrm{C}$ genotype 1. Eur J Clin Microbiol Infect Dis 2011;30:761-6.

[2] Kikuchi H, Katsuramaki T, Kukita K, Taketani S, Meguro M, Nagayama M, et al. New strategy for the antifibrotic therapy with oral administration of FR260330 (a selective inducible nitric oxide synthase inhibitor) in rat experimental liver cirrhosis. Wound Repair Regen 2007;15:881-8.

[3] Gong Y. Identifying the targets for treatment of liver fibrosis and hepatocellular carcinoma from both Western medicine and Chinese medicine. Chin J Integr Med 2012;18:245-9.

[4] Ba T, Bai WF, Wang H. [Clinical Observation on Treatment of Chronic Beta Particle Hepatitis with Two Kinds of Mongolian Medicine already Prepared by a Pharmacy for Main Dose of a Synthetical]. Liaoning Journal of Traditional Chinese Medicine 2004;32:391.

[5] Hepatology of Chinese Medical Association. [Infectious Diseases credits will. Guide to prevention of chronic hepatitis B]. Journal of Hepatology 2005;12:881-9. 
[6] The China Association of Integrative Medicine Professional Committee of liver disease. [Integrative Medicine of liver fibrosis Guide]. Integrative Medicine on Liver Diseases 2006;16:316-20.

[7] Zheng XY. [The guiding principles of Chinese medicine clinical research (for trial implementation)]. Beijing: Chinese Medical Science and Technology Press. 2002;44-150.

[8] Tanikawa K. Serum marker for hepatic fibrosis and related liver pathology. Pathol Res Pract 1994;190:960-8.

[9] Yao XX, Jiang SL, Tang YW, Yao DM, Yao X. Efficacy of Chinese medicine Yi-gan-kang granule in prophylaxis and treatment of liver fibrosis in rats. World J Gastroenterol 2005;11:2583-90.

[10] Hu YY, Cheng Y. [Comprehensive therapy of chronic hepatitis $\mathrm{B}$ induced liver fibrosis]. Zhongguo Zhong Xi Yi Jie $\mathrm{He} \mathrm{Za}$ Zhi 2008;28:765-8.

[11] Ba T, Zhao ZX, Zhang GW. [Mongolian medicine Dedu saffron the -7 flavor scattered on the protective effect of acute liver injury and acute toxicity pharmacological tests]. Liaoning Journal of Traditional Chinese Medicine 2006;33:494.

[12] Bai QY. [China Medical Encyclopedia (Mongolian Medicine)]. Shanghai: Shanghai Science and Technology Press. 1992;57.

[13] Health of the People's Republic of China Pharmacopoeia Committee. [The Ministry of Health of the People's Republic of China pharmaceutical standards Mongolian Medicine Volume]. 1998.
[14] Bai SY, Jason W, Wang AZ. [Expression of Serum IL 18 and Fas mRNA of Liver in Mice Hepatic Injury Model Induced by Dimethylnitrosamine]. Tianjin Traditional Chinese Medicine. 2003;31:787.

[15] Zhang JN, Zhao YY, Chen BL, et al. [Bulging piece hepatic fibrosis clinical observation]. Chinese Integrative stomach magazine 1994;3:156-7.

[16] Osakabe K, Ichino N, Nishikawa T, Sugiyama H, Kato M, Kitahara S, et al. Reduction of liver stiffness by antiviral therapy in chronic hepatitis B. J Gastroenterol 2011;46:132434.

[17] Xie DY, Lin BL, Xu QH, Chen YM, Lu WL, Li JG, et al. [The predictive value of ALT, HBeAg and HBV DNA levels at baseline and the degree of HBV suppression at week 12 adefovir dipivoxil treatment to the efficacy of it at week 52 in patients with $\mathrm{HBeAg}$-positive chronic hepatitis B]. Zhonghua Gan Zang Bing Za Zhi 2008;16:341-4.

[18] Zidek Z, Kmonickova E, Holy A. Cytotoxicity of pivoxil esters of antiviral acyclic nucleoside phosphonates: adefovir dipivoxil versus adefovir. Biomed Pap Med Fac Univ Palacky Olomouc Czech Repub 2005;149:315-9.

[19] Tong MJ, Hsu L, Chang PW, Blatt LM. Evaluation of current treatment recommendations for chronic hepatitis B: a 2011 update. J Gastroenterol Hepatol 2011;26:829-35.

[20] Muriel P, Rivera-Espinoza Y. Beneficial drugs for liver diseases. J Appl Toxicol 2008;28:93-103. 